\title{
Erbium-doped GaN epilayers synthesized by metal-organic chemical vapor deposition
}

\author{
C. Ugolini, N. Nepal, J. Y. Lin, and H. X. Jiang ${ }^{\text {a) }}$ \\ Department of Physics, Kansas State University, Manhattan, Kansas 66506
}

J. M. Zavada

U.S. Army Research Office, Durham, North Carolina 27709

(Received 20 July 2006; accepted 26 August 2006; published online 9 October 2006)

\begin{abstract}
The authors report on the synthesis of Er-doped GaN epilayers by in situ doping by metal-organic chemical vapor deposition (MOCVD). The optical and electrical properties of the Er-doped GaN epilayers were studied by photoluminescence (PL) spectroscopy and van der Pauw-Hall method. Both above and below band gap excitation results in a sharp PL emission peak at $1.54 \mu \mathrm{m}$. In contrary to other growth methods, MOCVD grown Er-doped GaN epilayers exhibit virtually no visible emission lines. A small thermal quenching effect, with only a $20 \%$ decrease in the integrated intensity of the $1.54 \mu \mathrm{m}$ PL emission, occurred between 10 and $300 \mathrm{~K}$. It was found that $\mathrm{Er}$ incorporation has very little effect on the electrical conductivity of the GaN epilayers and Er-doped layers retain similar electrical properties as those of undoped GaN. () 2006 American Institute of Physics. [DOI: 10.1063/1.2361196]
\end{abstract}

Incorporation of rare-earth atoms into a semiconductor host has received much attention due to the potential applications of rare-earth-doped materials as efficient optical amplifiers and light emitters. A majority of the research done for rare-earth doping has been devoted to the element $\mathrm{Er}^{1-16}$ since one of its intra- $4 f$ transitions occurs at a wavelength of $1.54 \mu \mathrm{m}$, corresponding to the minimum loss in silica fibers for optical communications. The problem with $\mathrm{Er}^{3+}$ emission in many semiconductors is the low emission efficiency at room temperature. However, it has been well recognized that the thermal quenching of the $\mathrm{Er}^{3+}$ emission is decreased significantly in wide band gap semiconductors. ${ }^{4}$ It has also been suggested that the neighboring environment created by more ionic host semiconductors increases the intensity of intra- $4 f$ $\mathrm{Er}^{3+}$ transitions. ${ }^{1,5}$ In light of these properties, the GaN material system appears to be an optimum host for Er since it has a band gap of $3.4 \mathrm{eV}$ and an electronegativity difference of -1.2 .

Much work has been dedicated to the incorporation of Er into GaN by methods such as ion implantation, hydride vapor phase epitaxy, metal-organic molecular beam epitaxy, and molecular beam epitaxy (MBE) ${ }^{5-16}$ There have been reports of Er incorporation, leading to devices, such as light emitting diodes (LEDs), that produce wavelengths ranging from the visible to infrared (IR). ${ }^{6,11,14}$ But all such devices suffer either from strong emission lines in the visible region and/or a low quantum efficiency at the IR wavelengths, severely limiting their prospects for practical devices for telecommunication systems. Until now, Er-doped GaN obtained by in situ incorporation by metal-organic chemical vapor deposition (MOCVD) has not been experimentally realized, even though devices, such as blue/green LEDs, based upon MOCVD synthesized GaN are among the highest quality. ${ }^{17}$ The difficulty in producing Er-doped GaN by the MOCVD process stems from the low vapor pressures of the Er precursors and/or the large organic chains, inherent in the Er pre-

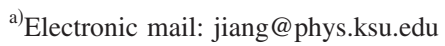

cursors containing elements that are parasitic to the quality of $\mathrm{GaN}$.

In this work, we report on the in situ incorporation of Er into GaN epilayers by MOCVD and their electrical and optical properties. The Er-doped GaN epilayers were synthesized by MOCVD in a horizontal reactor. All samples were grown on (0001) sapphire substrates. The growth of these epilayers began with a thin GaN buffer layer, followed by a $1.2 \mu \mathrm{m}$ GaN epilayer template and a $0.5 \mu \mathrm{m}$ Er-doped GaN layer. The growth temperature of the GaN template and Er-doped GaN layer was $1040^{\circ} \mathrm{C}$. An UV photoluminescence (PL) spectroscopy system was employed to study the optical properties of the Er-doped GaN. The PL system consists of a frequency doubled and tripled, $100 \mathrm{fs}$ Ti:sapphire laser operating at an average power of $150 \mathrm{~mW}$ at 263 and $395 \mathrm{~nm}$ and a repetition rate of $76 \mathrm{MHz}$. IR detection was accomplished by an InGaAs detector, while visible PL detection was accomplished by a multichannel plate photomultiplier tube, in conjunction with a $1.3 \mathrm{~m}$ monochromator, and a typical PL spectrum is depicted in Fig. 1. Room temperature transport properties of these films were measured by the

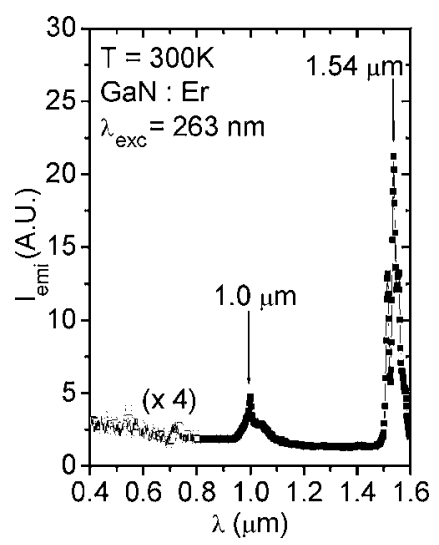

FIG. 1. Room temperature PL spectra of Er-doped GaN with laser excitation wavelength $\lambda_{\text {exc }}=263 \mathrm{~nm}$. The visible part of the spectrum is scaled by a factor of 4 . 


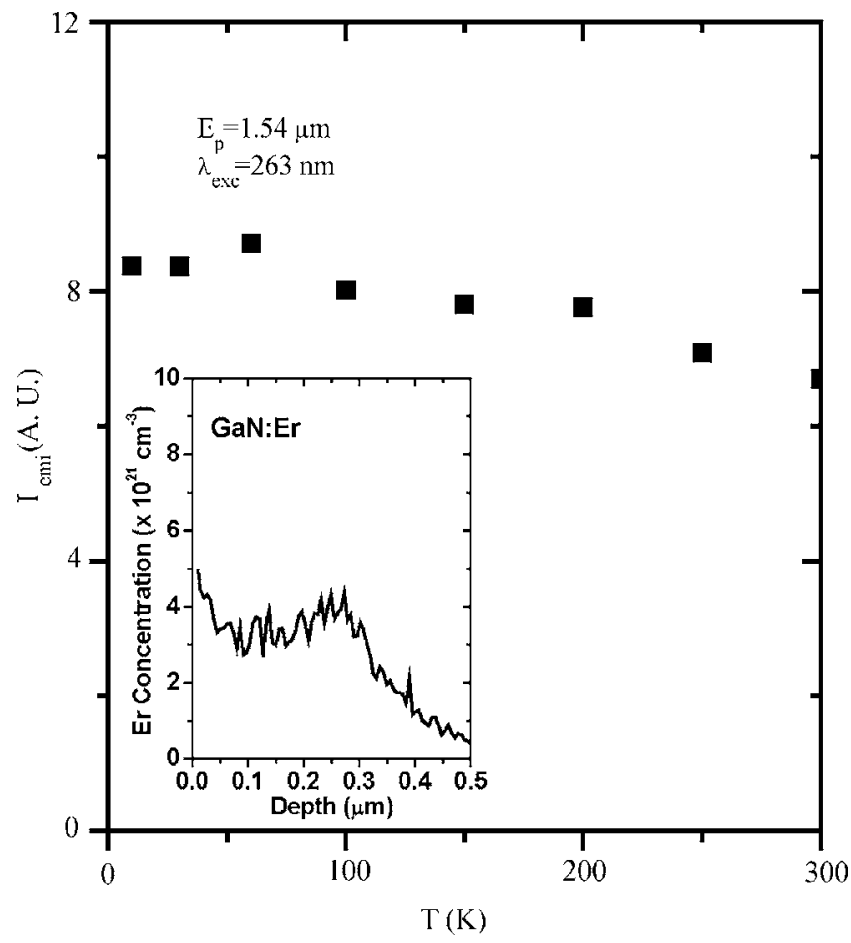

FIG. 2. Integrated PL emission intensity of the $1.54 \mu \mathrm{m}$ peak vs sample temperature with laser excitation wavelength $\lambda_{\mathrm{exc}}=263 \mathrm{~nm}$. The inset is the Er concentration profile of an Er doped $\mathrm{GaN}$ epilayer as determined by SIMS.

van der Pauw-Hall method. Secondary-ion mass spectroscopy (SIMS) was performed by the Evans Analytical Group to profile the Er concentration. The inset of Fig. 2 shows the Er profile for one of our Er-doped GaN epilayers as probed by SIMS.

Figure 1 is a room temperature PL spectrum of Er-doped GaN covering wavelengths from the visible to the IR region, for an excitation wavelength $\left(\lambda_{\text {exc }}\right)$ of $263 \mathrm{~nm}$. Two sharp emission peaks are observed at wavelengths of 1.0 and $1.54 \mu \mathrm{m}$ (with full widths at half maximum of 10.2 and $11.8 \mathrm{~nm}$ ), corresponding to the radiative intra- $4 f \mathrm{Er}^{3+}$ transitions from the ${ }^{4} I_{11 / 2}$ (second excited state) and the ${ }^{4} I_{13 / 2}$ (first excited state) to the ${ }^{4} I_{15 / 2}$ (ground state), respectively. The room temperature PL intensity of the $1.54 \mu \mathrm{m}$ peak is much larger than that of the $1.0 \mu \mathrm{m}$ emission line. Figure 1 also shows that virtually no visible emission lines can be observed in Er-doped GaN epilayers grown by MOCVD. This is in sharp contrast to the results of Er-doped GaN obtained by MBE or ion implantation, which exhibit the dominant intra- $4 f \mathrm{Er}^{3+}$ transitions to be from the ${ }^{2} H_{11 / 2}(537 \mathrm{~nm})$ and ${ }^{4} S_{3 / 2}(558 \mathrm{~nm})$ to the ${ }^{4} I_{15 / 2}$ (Refs. 12 and 15) ground state. In Fig. 2 is shown the high degree of thermal stability of the $\mathrm{Er}^{3+}$ emission. The integrated PL intensity of the $1.54 \mu \mathrm{m}$ emission decreases between 10 and $300 \mathrm{~K}$ by only $20 \%$. This low degree of thermal quenching is in good agreement with previous results of Favennec et al. ${ }^{4}$ and represents the lowest reported value for Er-doped GaN. ${ }^{9}$ These results clearly demonstrate the high potential of MOCVD grown Er-doped $\mathrm{GaN}$ epilayers for optical communication devices.

In Fig. 3 is a comparison of the PL spectra of Er-doped $\mathrm{GaN}$ at $10 \mathrm{~K}$, with above band gap excitation $\left(\lambda_{\text {exc }}=263 \mathrm{~nm}\right)$ and below band gap excitation $\left(\lambda_{\text {exc }}=395 \mathrm{~nm}\right)$. It is observed that the PL intensity of the $1.54 \mu \mathrm{m}$ emission with $\lambda_{\text {exc }}=263 \mathrm{~nm}$ excitation is ten times Downloaded 12 Jul 2010 to 129.118 .86 .45 . Redistribution subject

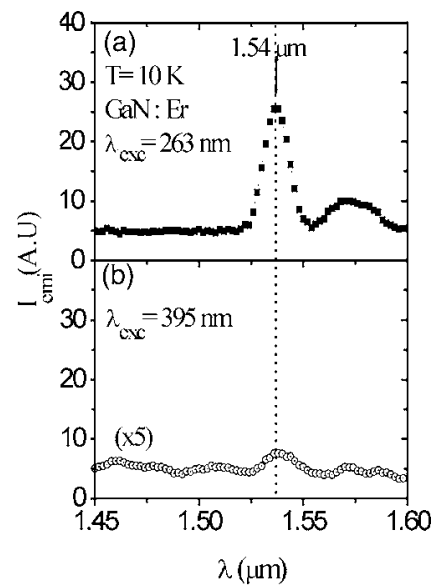

FIG. 3. PL spectra of Er-doped GaN for two different excitation wavelengths at $10 \mathrm{~K}\left(\lambda_{\mathrm{exc}}=263 \mathrm{~nm}\right.$ and $\left.\lambda_{\mathrm{exc}}=395 \mathrm{~nm}\right)$.

larger than that with $\lambda_{\mathrm{exc}}=395 \mathrm{~nm}$. It is apparent that the above band gap excitation is much more efficient for the $1.54 \mu \mathrm{m}$ emission. This result indicates that the generation of electron-hole $(e-h)$ pairs results in a more efficient energy transfer to Er centers than through defect centers in the epilayers. ${ }^{16}$ Thus, optical amplifiers and emitters operating at $1.54 \mu \mathrm{m}$ may be more efficient by employing an above band gap excitation scheme.

An important consideration for the realization of practical optoelectronic devices is the effect of Er incorporation on the electrical properties of GaN. Doping of GaN with Er may introduce defects that are a detriment to the required electrical conductivity. In Fig. 4, we plot optical and electrical properties (integrated PL emission intensity of the $1.54 \mu \mathrm{m}$ peak, resistivity, electron mobility, and free electron concentration) of Er-doped GaN epilayers as a function of $\mathrm{Er}$ concentrations between $(0.2$ and 1$) \times 10^{21} \mathrm{~cm}^{-3}$. The electrical properties were determined by Hall effect measurement. As can be seen in Fig. 4, the electron mobility, concentration, and resistivity are relatively unchanged

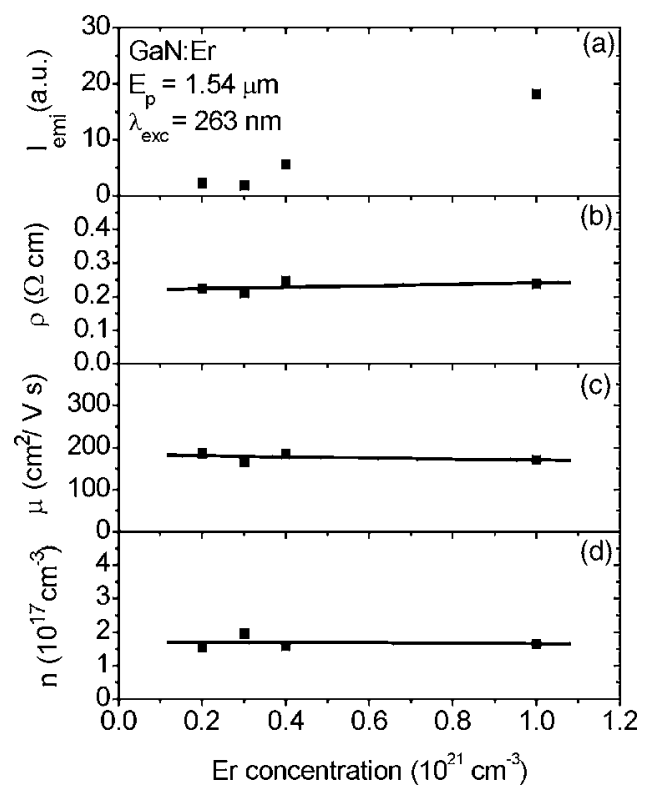

FIG. 4. Optical and electrical properties of epilayers as a function of Er concentration: (a) integrated PL emission intensity of the $1.54 \mu \mathrm{m}$ peak, (b) resistivity $\rho$, (c) electron mobility $\mu$, and (d) free electron concentration $n$.
AIP license or copyright; see http://apl.aip.org/apl/copyright.jsp 
with an increase in Er concentration. In fact, the values of mobility $\left(\sim 200 \mathrm{~cm}^{2} / \mathrm{V} \mathrm{s}\right)$, electron concentration $\left(\sim 2 \times 10^{17} \mathrm{~cm}^{-3}\right)$, and resistivity $(\sim 0.2 \Omega \mathrm{cm})$ are comparable to those of high-quality undoped MOCVD GaN $\left(400 \mathrm{~cm}^{2} / \mathrm{V} \mathrm{s}, 2 \times 10^{17} \mathrm{~cm}^{-3}, 0.05 \Omega \mathrm{cm}\right)$. The only noticeable difference is the smaller mobility of the Er-doped samples. These results can be explained if we consider the bonding nature of Er within a GaN host. It has been reported that the majority of Er atoms occupy the Ga site within the hexagonal wurtzite structure. ${ }^{1}$ It is also known that the normal charge state of bonding of Er inside an ionic host is $\mathrm{Er}^{3+}, 18,19$ which is the same as that of Ga inside the hexagonal wurtzite structure. This implies that the incorporation of Er centers into $\mathrm{GaN}$ is essentially isoelectronic doping and should not affect the free electron concentration. However, Er atoms will act as scattering centers, thereby reducing the electron mobility. Depicted in Fig. 4(a) is a plot of the integrated PL intensity of the $1.54 \mu \mathrm{m}$ emission versus $\mathrm{Er}$ concentration with $\lambda_{\text {exc }}=263 \mathrm{~nm}$. The emission intensity of the $1.54 \mu \mathrm{m}$ peak does increase with increasing $\mathrm{Er}$ concentration.

In summary, we have synthesized Er-doped GaN epilayers with in situ doping by MOCVD. PL results showed that both above and below band gap excitation produces emission at $1.54 \mu \mathrm{m}$ whose intensity increases with higher Er concentration. The Er emission at $1.54 \mu \mathrm{m}$ in GaN has a high degree of thermal stability; the decrease in emission intensity between 10 and $300 \mathrm{~K}$ was only about $20 \%$. PL results also indicated that above band gap excitation is much more efficient for $\mathrm{Er}^{3+}$ emission at $1.54 \mu \mathrm{m}$. Hall effect measurements revealed that Er-doped GaN epilayers have comparable electrical properties to those of high-quality undoped GaN. These results, together with the absence of virtually any visible PL emission lines, demonstrate the very high potential of these materials for applications in optoelectronic devices operating at the main telecommunication wavelength of $1.54 \mu \mathrm{m}$.
This work is supported by ARO under Grant No. W911NF-05-1-0196.

${ }^{1}$ J. M. Zavada and Dahua Zhang, Solid-State Electron. 38, 1285 (1995).

${ }^{2}$ M. R. Brown, A. F. J. Cox, W. A. Shand, and J. M. Williams, Advances in Quantum Electronics 2, 69 (1974).

${ }^{3}$ H. Ennen, J. Schneider, G. Pomrenke, and A. Axmann, Appl. Phys. Lett. 43, 943 (1983).

${ }^{4}$ P. N. Favennec, H. L'Haridon, M. Salvi, D. Moutonnet, and Y. LeGuillou, Electron. Lett. 25, 718 (1989).

${ }^{5}$ R. G. Wilson, R. N. Schwartz, C. R. Abernathy, S. J. Pearton, N. Newman, M. Rubin, T. Fu, and J. M. Zavada, Appl. Phys. Lett. 65, 992 (1994).

${ }^{6}$ J. T. Torvik, R. J. Feuerstein, J. I. Pankove, C. H. Qiu, and F. Namavar, Appl. Phys. Lett. 69, 2098 (1996).

${ }^{7}$ S. Kim, S. J. Rhee, D. A. Turnbull, E. E. Reuter, X. Li, J. J. Coleman, and S. G. Bishop, Appl. Phys. Lett. 71, 231 (1997).

${ }^{8}$ D. M. Hansen, R. Zhang, N. R. Perkins, S. Safvi, L. Zhang, K. L. Bray, and T. F. Kuech, Appl. Phys. Lett. 72, 1244 (1998).

${ }^{9}$ J. D. MacKenzie, C. R. Abernathy, S. J. Pearton, U. Hömmerich, J. T. Seo, R. G. Wilson, and J. M. Zavada, Appl. Phys. Lett. 72, 2710 (1998).

${ }^{10}$ A. J. Steckl, M. Garter, R. Birkhahn, and J. Scofield, Appl. Phys. Lett. 73, 2450 (1998).

${ }^{11}$ M. Garter, J. Scofield, R. Birkhahn, and A. J. Steckl, Appl. Phys. Lett. 74, 182 (1999).

${ }^{12}$ R. H. Birkhahn, R. Hudgins, D. S. Lee, B. K. Lee, A. J. Steckl, A. Saleh, R. G. Wilson, and J. M. Zavada, MRS Internet J. Nitride Semicond. Res. 4S1, G3.80 (1999).

${ }^{13}$ A. J. Steckl, J. Heikenfeld, M. Garter, R. Birkhahn, and D. S. Lee, Compound Semicond. 6, 48 (2000).

${ }^{14}$ J. M. Zavada, S. X. Jin, N. Nepal, H. X. Jiang, J. Y. Lin, P. Chow, and B. Hertog, Appl. Phys. Lett. 84, 1061 (2004).

${ }^{15}$ C. S. Son, S. Kim, Y. H. Kim, I. K. Han, Y. T. Kim, A. Wakahara, I. H. Choi, and H. C. Lopez, J. Korean Phys. Soc. 45, 955 (2004).

${ }^{16}$ X. Wu, U. Hommerich, J. D. MacKenzie, C. R. Abernathy, S. J. Pearton, R. Schwartz, R. G. Wilson, and J. M. Zavada, Appl. Phys. Lett. 70, 2126 (1997).

${ }^{17}$ Shuji Nakamura and Gerhard Fasol, The Blue Laser Diode: GaN Based Light Emitters and Lasers (Springer, Berlin, 1997), pp. 201-216.

${ }^{18}$ B. Wybourne, Spectroscopic Properties of Rare Earths (Interscience, New York, 1965), pp. 3-8.

${ }^{19} \mathrm{~S}$. Hufner, Optical Spectra of Transparent Rare Earth Compounds (Academic, New York, 1978), pp. 14-16. 